\title{
La adolescencia en contexto: Representaciones Sociales en adolescentes de Medellín y Armenia, Colombia
}

\author{
Adolescence in a context: social representations in \\ adolescents from Medellín and Armenia (Colombia)
}

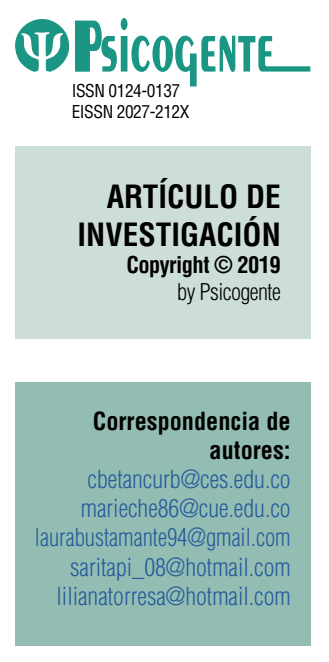

Recibido: $20-10-17$ Aceptado: $16-08-18$ Publicado: 01-07-19

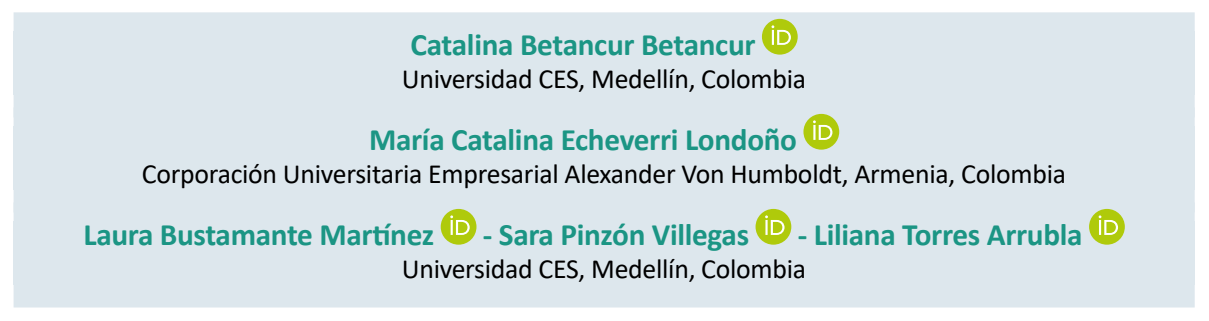

Resumen

Objetivo: Comprender las representaciones sociales de adolescencia en adultos y adolescentes de las ciudades de Medellín y Armenia.

Método: Investigación cualitativa desde el enfoque procesual de la teoría de las representaciones sociales. La investigación se llevó a cabo en dos momentos: uno exploratorio y otro de profundización. Se realizó una evocación libre de palabras para identificar el núcleo central y los sistemas periféricos de la representación; a partir de esto, se realizaron grupos focales y entrevistas semiestructuradas que permitieron ampliar el horizonte de significado de la representación e identificar los procesos de objetivación y anclaje. En la investigación participaron 298 adolescentes habitantes de Medellín y Armenia.

Resultados: El núcleo central para Armenia está constituido por la palabra "etapa", mientras que, en Medellín, se encuentra la "responsabilidad". Aunque en lo nuclear la representación en ambas ciudades comparte múltiples elementos, hay una diferencia fundamental respecto a la perspectiva de futuro, pues mientras para los adolescentes de Medellín es posible proyectar la vida en la adultez, para los adolescentes de Armenia prima el tiempo presente y no hay una delimitación del fin de la adolescencia.

Conclusiones: La representación social de la adolescencia se ordena en torno a la idea de transición acompañada por diversos cambios; en este proceso, la reconfiguración de la relación con la familia y los amigos resulta fundamental. La experiencia de la adolescencia no es universal, pues el estrato socioeconómico, el nivel económico de la ciudad en que se vive y las posibilidades de desarrollo que ofrece, da un carácter diferencial al modo en que es significada y vivida.

Palabras clave: representaciones sociales, actitudes adolescentes, adolescencia

Abstract

Propose: Figure out social representations during adolescence in adults and teenagers from Medellín and Armenia (Colombia, South America).

Method: A qualitative research study was conducted based on a theory of social representations. This research study was developed in two stages: an exploratory stage and the other, a deepening stage. In the exploratory stage, free evocation of words in order to identify a core and peripheral elements of social representations were produced. In the second stage, focus groups and semi-structured interviews to allow an expansion of meanings and to identify objectification and setting processes. The research involved 298 adolescents from Medellín and Armenia.

Results: The core social representation for Armenia, is suggested by the word "stage", while, in Medellín, is "responsibility". Although, taking into account that there are many shared elements in relation with core social representations corresponding to each city, there is a fundamental difference related to a future

Cómo citar este artículo (APA):

Betancur Betancur, C., Echeverry Londoño, M.C. , Bustamante Martínez, L.,Pinzón Villegas, S.\& Torres Arrubla, L. (2019). La adolescencia en contexto:

Representaciones Sociales en adolescentes de Medellín y Armenia, Colombia. Psicogente 22(42), 1-25. https://doi.org/10.17081/psico.22.42.3471 
perspective; this is because while for adolescents in Medellín is possible to project life into adulthood, for teenagers from Armenia present time prevails and there is no delimitation for the end of adolescence.

Conclusions: Social representations related to adolescence is organized around the idea of transition with multiple changes. In this process, the relationship with family and friends become fundamental. The experience of adolescence is not considered universal; it is because the adolescent's socioeconomic status, economic level of a city where those adolescents live and also, the possibilities for development offered in a specific context, give a differential character to the way adolescence is represented and experienced.

Key words: social representations, adolescent attitudes, adolescence

\section{INTRODUCCIÓN}

La adolescencia ha sido definida como un periodo de transición del desarrollo entre la niñez y la adultez; cronológicamente estaría ubicada entre los 13 y 19 años aproximadamente (Papalia, Olds, de León \& Villamizar, 1997; McKinney, Fitzgerald \& Strommen, 1993; Santrock, 2004) y se define por unos cambios físicos, psicológicos y sociales (Tesouro i Cid, Palomanes Espadalé, Bonachera \& Fernández, 2013). Según Silva (2007), "como constructo cultural la adolescencia es generalmente definida como un periodo biopsicosocial entre los 10 y 20 años, aproximadamente. Es una etapa en la que tienen lugar importantes modificaciones corporales, así como de adaptación a nuevas estructuras psicológicas y ambientales que conducen de la infancia a la adultez" (p.12), lo cual coincide con las teorías clásicas de la adolescencia, que la tipifican como una etapa de preparación para la vida adulta, entendida como el momento de plena integración a la sociedad; en este periodo, se pasa de la inmadurez a la madurez, de la incapacidad a la capacidad, y de la irresponsabilidad a la responsabilidad, trayectoria que marca lo que la psicología considera como el desarrollo normal (Pavez, 2012); por ejemplo, Limones (2011) afirma que "la adolescencia es un concepto mucho más extenso donde el crecimiento y el desarrollo evolutivo todavía no están completos" (p.235).

Aun cuando no hay un orden universal para la clasificación de esta población según rango de edad, sí es posible afirmar que la existencia de la adolescencia se da por sentada y que frente a ella se suponen unas características homogéneas y universales (Brunet \& Pizzi, 2013); estas son conocidas como estereotipos generacionales, pues de acuerdo con la edad, se establecen en las sociedades derechos y deberes que configuran una normativa social (Pavez, 2012). Al respecto, y considerando que lo único que tienen los adolescentes en común es la edad, diversos autores afirman que el concepto de adolescencia es una construcción social que está atravesada por cuestiones morales, ideológicas e incluso políticas, que hacen de la vivencia de la adolescencia un asunto diferenciado según el contexto (Margulis, 2004a; Taguenca, 2016): 
Hay muchos modos de experimentar la adolescencia: las formas en que se experimentan los años de crecimiento y madurez corporal están profundamente influidas y condicionadas por la cultura, la época, el género y la diferenciación social. Cada sociedad, cada época, cada sector social, construye las formas culturales e institucionales que inciden en esta etapa de la vida. (Margulis, 2004b, p.2)

De acuerdo con Alpízar y Bernal (2003), en torno a la adolescencia hay discursos y prácticas producidas y reproducidas por múltiples instituciones, que muestran los miedos, la nostalgia y la idealización por parte de los adultos de una etapa que consideran tan extraña como familiar. Dichas posturas se caracterizan, entre otras cosas, por ser homogeneizantes, adultocéntricas y estigmatizantes, lo que ubica a los adolescentes en un lugar desfavorable en el entramado social.

Si se define la realidad como construcción, es posible entonces reelaborar, reconstruir y deconstruir los discursos y, en consecuencia, las prácticas frente a los adolescentes. Una de las vías para identificar los modos en que la adolescencia es significada en un colectivo específico, es la teoría de las representaciones sociales, pues estas hablan del saber del sentido común que se construye en torno a un objeto en el marco de las interacciones, posibilitando dotar de sentido a la realidad social y actuar sobre ella (Araya, 2002; Moscovici, 1979).

Por su parte, Jodelet (1986) afirma que las representaciones sociales son una forma de pensamiento social y práctico que da lugar a una modalidad de conocimiento particular, el saber del sentido común, destacando dos elementos principales, uno, como formas de conocimiento que tienen un carácter práctico y dos, como un concepto que abre la pregunta sobre el proceso de formación.

Es el enfoque procesual (Banchs, 2000) aquel que se interesa por los procesos de formación de la RS, para lo cual recurre a dos conceptos: el anclaje, definido como un proceso que permite organizar el mundo en términos de la clasificación y asimilación de objetos y fenómenos dentro de representaciones y categorías sociales; como segunda función se encuentra la objetivación, que se define como un proceso multifacético a través del cual el conocimiento representado socialmente adquiere su forma específica (Wagner, Hayes \& Flores, 2011). Las representaciones sociales son producidas por la experiencia 
cotidiana, por lo que la adolescencia se constituye en objeto posible de representación (Materán, 2008).

La investigación titulada Representaciones sociales de la adolescencia, construidas por adolescentes y adultos en las ciudades de Medellín y Armenia, se propuso como uno de sus objetivos comprender cómo se significa y se experimenta la adolescencia, desde los mismos adolescentes, en dos ciudades de Colombia, a fin de analizar si las características de ambas ciudades producen representaciones diferenciadas de la adolescencia, apoyando así el supuesto según el cual esta, más que una etapa, es una construcción social atravesada por las condiciones históricas, sociales, económicas y políticas de los contextos.

En este sentido, es fundamental tener en cuenta que Armenia es la capital del departamento del Quindío, conocida por ser una de las principales ciudades del eje cafetero en Colombia; cuenta con una población aproximada de 298 mil habitantes. Esta ciudad es Ilamada por muchos "la ciudad milagro" debido a su rápido crecimiento en un corto periodo de tiempo y después de haber sido afectada por el terremoto de 1999 en el que hubo alrededor de 1.230 muertos, 5.300 heridos y alrededor de 50.000 edificaciones afectadas (Carrillo, De la Rosa Bobadilla \& Gutiérrez, 2014).

Debido a este terremoto se impulsaron procesos de urbanización y agro-turísticos que dieron a la región un auge con respecto al alojamiento rural y fincas cafeteras que se dedican igualmente a esta labor (Gobierno del Quindío, 2012). En el departamento del Quindío, el 19,2 \% son niños y niñas (0 a 12 años) y el 29,4 \% son adolescentes y jóvenes (12-29 años) donde el $34 \%$ corresponde a los adolescentes (12 a 18 años); la ciudad de Armenia concentra el $44,5 \%$ de los niños, niñas y adolescentes del departamento (Gobernación del Quindío, 2016).

Desde el Diagnóstico Social Situacional de los niños, niñas, jóvenes, adolescentes y sus familias, el departamento del Quindío se ha planteado garantizar un adecuado desarrollo psicosocial, físico y mental de los niños, niñas y adolescentes; desde el año 2012 se ha estado planteando la necesidad de reconocer el papel protagónico y estratégico de los adolescentes en la construcción de proyectos que propendan por su desarrollo psicosocial óptimo y la atención oportuna de sus necesidades (Alcaldía de Armenia, 2014; Gobernación del Quindío, 2016). 
La política pública de juventud de Armenia busca establecer los marcos políticos e institucionales para garantizar los derechos básicos de los jóvenes tales como educación, cultura y libre desarrollo de la personalidad. Buscando que la política fuera incluyente, se crearon unos espacios en los cuales participaron el consejo de juventud, personeros estudiantiles y líderes juveniles; la formulación de la política estuvo orientada desde un enfoque de derechos y la expansión de capacidades tanto individuales como colectivas, permitiendo la construcción de realidades a partir de los mundos simbólicos y las posibilidades de diálogos intergeneracionales con las entidades públicas y privadas (Alcaldía de Armenia, 2014).

Por su parte, Medellín es un municipio en el Valle de Aburrá que pertenece al departamento de Antioquia. En contraposición con el modelo de Armenia, el económico de industrialización y modernización de Medellín la reconocen hoy como una de las ciudades más importantes de Colombia a nivel educativo, social y económico, por su habilidad para reducir indicadores de analfabetismo y pobreza, así como su capacidad para innovar y enfrentar el terrorismo del que fue víctima en la década de los 80 y 90 del siglo pasado a manos del cartel de Medellín liderado por Pablo Escobar (Schindler Andino, 2013).

En Medellín, el perfil demográfico para el año 2017, estima que 325.266 personas están entre los 10 y 19 años, lo que corresponde aproximadamente al 13 \% de la población. (Alcaldía de Medellín, 2015). El actual plan de desarrollo, contiene 30 proyectos dirigidos a población hasta los 17 años, que buscan mejorar la educación, salud y protección de los derechos (Secretaría del Concejo Municipal, 2016); si a esto se suma que la ciudad cuenta con políticas públicas y secretaría en juventud (Consejo Municipal de Política Social y de Equidad, 2014) -que abarca desde los 14 hasta los 28 años, incluyendo el rango de edad que se entiende por adolescencia-, es posible afirmar que para Medellín los adolescentes son una prioridad.

A través del acuerdo número 019 de 2014, por medio del cual se actualiza y adopta la política pública de juventud en Medellín, se establece el marco que permite garantizar el reconocimiento, inclusión, participación, equidad y goce efectivo de derechos, por medio de la promoción de espacios, movimientos, alianzas y entornos que favorezcan el desarrollo integral y la comprensión de dinámicas y realidades de la población juvenil en la ciudad (Alcaldía de Medellín, 2014). 
Como se mencionó anteriormente, ambas ciudades desarrollaron una política pública para garantizar los derechos de los jóvenes, lo cual muestra un panorama distinto al que comúnmente se encuentra en las investigaciones sobre adolescencia, en las cuales se ha abordado desde una perspectiva problemática y de crisis. Algunas de estas temáticas son el sexo sin protección, el embarazo adolescente (Blázquez, 2012), la drogadicción (Becoña, 2000; González \& Andrade, 2012), la delincuencia, la violencia (Acero, Escobar \& Castellanos, 2007; Alba, 2010; Andrade, 2012; Toro, Paniagua, González \& Montoya, 2009), los trastornos mentales relacionados con la imagen corporal, la alimentación y el suicidio (Deossa, Restrepo \& Velásquez, 2015; Loboa \& Morales, 2013); y los conflictos con los padres y adultos en general (Luna \& Cruz, 2014).

La proliferación de estudios sobre la adolescencia desde estos referentes ha tenido como consecuencia la perpetuación de imágenes estereotipadas que se asocian con actitudes hacia los adolescentes, marcadas por la necesidad de control y el prejuicio (Ridao \& Moreno, 2008); desde este punto, las apuestas políticas para relevar el lugar del adolescente como actor social y constructor de realidades, pueden tener dificultades a la hora de su implementación. Por tal motivo, y considerando además que la adolescencia, en tanto concepto y experiencia, es socialmente construida, es necesario adelantar investigaciones que procuren conocer los sentidos y significados que las personas le otorgan desde su vida cotidiana (Chaves, 2005), lo cual implica considerar las especificidades de los contextos en los que la adolescencia tiene lugar.

\section{MÉTODO}

\subsection{Diseño}

Se realizó una investigación cualitativa, que considera la realidad como una construcción mediada por la historia, y que permite estudiarla desde la posición particular de sus protagonistas, es decir, desde una perspectiva contextualizada, teniendo en cuenta sus relaciones y dinámicas, dándole importancia a la interpretación de los fenómenos de acuerdo con lo que cada hecho significa para las personas (Denzin \& Lincoln, 2011).

\subsection{Participantes}

Participaron 298 adolescentes, 148 habitantes de la ciudad de Armenia, y 150, de Medellín; en ambas ciudades se contó con hombres y mujeres, de diferentes edades, niveles socioeconómicos y comunas. 
Tabla 1.

Características sociodemográficas de los participantes

\begin{tabular}{ccc}
\hline VARIABLES & ARMENIA & MEDELLÍN \\
\hline & EDAD & \\
\hline $12-15$ & 102 & 90 \\
$16-18$ & 46 & 60 \\
\hline & SEXO & 61 \\
\hline Hombres & 58 & 89 \\
\hline Mujeres & 90 & \\
\hline $1-2$ & ESTRATO SOCIOECONÓMICO & 66 \\
$3-4$ & 28 & 29 \\
$5-6$ & 83 & 55 \\
\hline & 37 & 24 \\
\hline Rural & COMUNA & 126 \\
\hline
\end{tabular}

Para ser considerados en la investigación, debían tener entre 12 y 18 años atendiendo a la definición del rango de edad para adolescencia estimado desde la normatividad colombiana (Congreso de Colombia, 2006); en el caso de los menores de edad, se contó con el consentimiento informado de los padres o representantes legales y, en todo caso, con el asentimiento de los participantes. Se realizó un muestreo a conveniencia (Salamanca \& MartínCrespo, 2007). La información sociodemográfica fue recopilada a través de una ficha de caracterización sociodemográfica que fue piloteada con 50 personas en cada ciudad que no fueron incluidas como participantes en la investigación; a partir de este pilotaje, se implementaron los ajustes requeridos en el cuestionario.

\subsection{Instrumentos}

Para la investigación se utilizaron diversos instrumentos; el primero, que hizo parte del momento exploratorio, fue una ficha de evocación de palabras en la cual se presentó a los participantes una hoja de papel con la palabra "adolescencia" escrita en la parte superior; los participantes debían escribir aquellas palabras que asociaran con el término indicado y luego jerarquizarlas según su importancia para entender la palabra "adolescencia".

Con base en el análisis de este momento, se generaron guías de grupos focales y entrevistas que buscaron profundizar en el contenido de las representaciones esbozado gracias a la evocación; a los participantes se les presentó el resultado de la evocación en una nube de palabras, y a partir de ellas se 
indagó por el significado de esas palabras y por la manera en que las consideraban relacionadas con la adolescencia; igualmente, se indagó acerca del inicio y el final de la adolescencia, sus características, las relaciones que en ella se establecen y las experiencias de ser adolescente.

Estos procesos narrativos se consideraron importantes en tanto se convierten en un espacio participativo para dimensionar los aspectos relacionados con el sentir, pensar y las vivencias de los participantes, lo cual lleva a la reflexión y explicación de experiencias que se recolectan a modo de datos cualitativos y que permite identificar la RS (Martínez, citado por Hamui-Sutton \& Varela-Ruiz, 2013). En los grupos focales y las entrevistas, participaron 32 adolescentes de los 298 vinculados al proceso en ambas ciudades.

\subsection{Procedimiento}

Se trabajó el enfoque procesual de la teoría de las representaciones sociales, que de acuerdo con Banchs (2000), se caracteriza por

considerar que para acceder al conocimiento de las representaciones sociales se debe partir de un abordaje hermenéutico, entendiendo al ser humano como productor de sentidos, y focalizándose en el análisis de las producciones simbólicas, de los significados, del lenguaje, a través de los cuales los seres humanos construimos el mundo en que vivimos (p.36).

El enfoque procesual hace uso de los conceptos de objetivación y anclaje; el primero, hace referencia al proceso mediante el cual ese objeto de conocimiento que es abstracto se cosifica, es decir, se despoja de toda la arbitrariedad simbólica que posee y se convierte en una imagen, que pueda ser usada por esa persona o grupo. Este proceso es llevado a cabo a su vez, según Jodelet (1986) a través de tres fases: la construcción selectiva, esquematización estructurante y la naturalización. La primera hace alusión a la extracción de ciertos elementos o características de ese objeto que resultan significativos a ese sujeto o grupo, y se descontextualizan; la segunda se refiere a la organización de esos elementos seleccionados en un esquema o núcleo figurativo que permite erigir la representación, y la última fase, remite a la imagen que permite concretizar y cosificar tal objeto.

El anclaje, como segundo proceso, es por medio del cual se integran esas nuevas representaciones al sistema representacional pre-existente en el sujeto o grupo, haciéndolas funcionales y útiles; asimismo, el proceso que 
conlleva la estructuración de las RS, permite realizar una diferenciación en cuanto a tres dimensiones que son consideradas de gran relevancia al tomar como forma de conocimiento este marco explicativo: la actitud, la información y el campo de representación (Araya, 2002).

Este marco de referencia orientó los dos momentos metodológicos que ordenaron la investigación; el primero, fue uno exploratorio, en el que se realizó una evocación libre de palabras (Araya, 2002; Vergés, 1992); en el segundo momento, de profundización, se realizaron entrevistas y grupos focales que posibilitaron el análisis de los procesos de objetivación y anclaje de la representación.

\subsection{Análisis de datos}

Luego de realizada la evocación, se transcribieron en un libro de Excel la totalidad de las palabras evocadas por cada participante, y posteriormente, se procesaron en el programa EVOC, que permite la identificación de las frecuencias y los rangos de evocación de cada una de las palabras; de tal modo, pudieron identificarse aquellas palabras más evocadas y con jerarquía en los primeros lugares, y fue con ellas que se elaboró la nube de palabras.

Por su parte, lo relatado en las entrevistas y los grupos focales fue transcrito y procesado en una matriz, que posibilitó la organización en categorías y su respectiva codificación; posterior a ello, se siguió el modelo propuesto por Bonilla y Rodríguez (1997), que consiste en la realización de dos tipos de análisis, uno descriptivo para poder pasar a un segundo que se basa en una interpretación de resultados. Esto último estuvo caracterizado por la generación de relaciones entre las categorías, para lo cual se recurrió a la generación de redes y taxonomías de organización, lo que permitió elaborar un mapa conceptual en el que se condensó el análisis realizado, y favoreció la búsqueda de los procesos de constitución de la RS.

\subsection{Conflicto de interés}

Los investigadores declaran que en el proceso de investigación no se presentaron conflictos de interés.

\section{RESULTADOS}

De acuerdo con la información generada en la investigación, se hará primero alusión al campo representacional constituido, lo cual implica reconocer la información y las actitudes que componen dicha representación (Moscovici, 
1963) y conforman el campo representacional; para dar cuenta de esto, se hará referencia a las categorías construidas a partir del análisis de la información. Es importante aclarar que en las dimensiones de información y actitud no se encuentran mayores diferencias en torno a lo que es y lo que se vive en la adolescencia, sin embargo, hay ciertos elementos contextuales que permiten diferenciar lo que se cree respecto a ella.

\subsection{De Niños a Adultos: la Transición de la Adolescencia}

En relación a la dimensión de la información, la adolescencia es concebida como ese periodo intermedio entre la niñez y la adultez; esto en Medellín toma el carácter de proceso de abandono de un tiempo previo y de preparación para uno nuevo, mientras que para los adolescentes de Armenia, este tiempo es algo "normal", es decir, que es una etapa atravesada por todas las personas y que lejos de ser problemática, es algo natural en la experiencia y similar al resto de etapas que se atraviesan en la vida. En ambas ciudades los adolescentes caracterizan a la niñez como un momento de inocencia frente al mundo y a la vida, mientras que la adolescencia sería un despertar a la realidad:

"Es como cuando empiezan a aparecer tantas cosas de las que uno no se había dado cuenta porque uno pequeño es muy inocente y no sabes, pues, como, en el mundo en el que vives". (Mujer adolescente, Medellín)

"Es una etapa normal como cualquier otra". (Hombre adolescente, Armenia)

La adolescencia también se caracteriza, según los participantes, por la exploración, lo cual lleva consigo cambios en vínculos, gustos y actividades; así mismo, se resaltan los cambios emocionales, definidos como altibajos en el estado de ánimo que son más frecuentes en las mujeres que en los hombres.

Tanto en Medellín como en Armenia, los cambios físicos son resaltados como los más evidentes, y hacen referencia a la voz, el crecimiento de los senos y el aumento de estatura; sin embargo, en Medellín mencionan que esto no define la adolescencia, sino que son características de ella; por su parte en Armenia, según los adolescentes, estos cambios son los responsables de impulsar las transformaciones a nivel emocional y psicológico. 
“[...] Primero, cambios corporales, eso es algo que primero se ve, pues empieza todo como a salir pelos en todos lados, principalmente lo que más he dicho, la voz". (Hombre adolescente, Medellín) Pues en mi caso son como cosas muy chistosas que pasan, pues no chistosas, serias, pero pues, hay... o sea, los adolescentes pasamos por el cambio de hormonas y todo eso, entonces hay unos que mantienen como muy... no sé... calenturientos y así. (Mujer adolescente, Armenia)

Los cambios psicológicos percibidos como parte de la adolescencia permiten vislumbrar la dimensión de la actitud; para los adolescentes de Armenia se traducen en la búsqueda de sí mismos, de vivir, experimentar y lograr el aprendizaje:

"Uno aprende de las cosas malas que apenas vamos a vivir, es mejor probar para saber, solo que lo malo es mejor, más rico, solo que de lo malo se aprenden más cosas. Si usted fuma usted sabe que es malo entonces usted lo va a dejar o si toma uno no sabe que es malo, pero si tú no lo haces, no vas a saber si es malo o es algo que necesitas para aprender". (Mujer adolescente, Armenia)

En Medellín, por su parte, se habla de cambios en la posición frente a la vida, de tener sueños, darse cuenta, perder la inocencia y cuestionarse:

"No creo que en la adultez como que cambie con eso, pero en la niñez sí porque, como te digo, de la adolescencia a la niñez puede haber un cambio total de percepción o de pensamientos, entonces sí puede haber como cierta... o alejarse ciertas costumbres o ciertos hábitos que sabes que no es bueno [sic] para ti". (Hombre adolescente, Medellín)

Siguiendo con la dimensión de la información, para los adolescentes en Medellín esta etapa no es algo cronológico, sino que es el momento en donde se consolidan los procesos y se construyen respuestas sobre el ser y el lugar en el mundo; para ellos, el fin de la adolescencia se relaciona con el logro de la responsabilidad, que se diferencia de lo que llaman "responsabilidades" y que se refieren específicamente a las obligaciones domésticas y escolares; la responsabilidad es la conquista fundamental en la adolescencia, y que se consolida en la consecución de un trabajo como vía para sostenerse a nivel económico y ser independiente en la toma de decisiones. De tal modo, puede suceder que un adolescente genere ingresos básicos sin que sea indepen- 
diente, lo que no implica adultez, o también podría suceder que alguien con edad adolescente, sea un adulto porque ya responde por sí mismo.

"Empezaría a perder su adolescencia, porque ya se preocupa es por los gastos, lo que tiene que hacer y lo que ya es. Digamos que mi hermanito, que tiene 16 años, que ya empiece a trabajar para independizarse, pues usted puede trabajar, pero para sus cosas, sus gustos, peor viviendo con la mamá y trabajando solo para cosas suyas; pero si ya empieza a trabajar y ya se quiere es ir de su casa y ya quiere vivir como solo y tiene su trabajo seguro por decirlo así, ya ahí sí perdería como su adolescencia". (Hombre adolescente, Medellín)

Por último, los participantes de Medellín afirman que la adolescencia culmina con la madurez, relacionada con volverse un ciudadano del mundo que aporta a la sociedad y se responsabiliza de sus propias decisiones:

"No es como que se vaya a acabar en una edad, pero yo sí digo que uno llega [sic] un punto en el que adquiere una madurez, pues, en el sentido en el que uno ya es un ciudadano del mundo, que uno es ya una persona para la sociedad, entonces ahí, es cuando ya uno empieza a ser un adulto, pero ser un adulto no significa que tú debas dejar esa libertad ni la diversión de la adolescencia, pues, eso también es una decisión muy personal. Entonces no es como algo que empieza y se acaba, sino que es como un proceso y uno decide cuando hacer esa transición". (Mujer adolescente, Medellín)

Por su parte, en Armenia el fin de la adolescencia llega cuando el adolescente se independiza y se va de la casa.

"No, pues yo creo que cuando uno se casa, con su peluche... con una mujer bien. Cuando tiene hijos". (Hombre adolescente, Armenia)

No obstante, esta temática no fue ampliamente abordada por los adolescentes en Armenia; se habla de la adolescencia como la etapa previa de la adultez, sin embargo, el ser adulto aún no está representado y, en consecuencia, la visión de futuro no está claramente consolidada.

\subsection{Quién soy y para dónde voy: Orientación de la construcción adolescente}

En ambas ciudades, se plantea la pregunta "¿quién soy y para dónde voy?" como aquella que ordena la experiencia del adolescente; no obstante, el 
proceso de responderla adquiere matices diferentes según el contexto y da muestras de la dimensión referente a la actitud. En Medellín la respuesta a esta pregunta, que está vinculada a la confusión, se supone resuelta en la adultez, y trae consigo una transformación en lo que se sueña para la vida, pasando de unos sueños materiales en la niñez, a unos trascendentales en la adolescencia:

"[...] que no sabe realmente qué le gusta o qué no, y no sabe qué hacer como con la vida, no sabe que está haciendo y por qué lo está haciendo o qué va a hacer en un futuro. Todavía no sabe, por ejemplo, qué va a estudiar, ehhh, sí". (Mujer adolescente, Medellín) "Los sueños también han cambiado mucho porque antes yo soñaba como con cosas materiales y ya sueño con ser profesional, ser una gran médica, ser una gran persona, ya mis sueños no son como tan materiales como, "ay, tener este celular", "tener esta ropa", no, mis sueños ya han cambiado". (Mujer adolescente, Medellín)

En Armenia, cuando los adolescentes tienen su encuentro con el mundo, surge la pregunta por lo que se quiere hacer en un futuro; están centrados en buscarse a sí mismos. En su mayoría, quieren continuar estudiando e independizarse de sus padres como meta última, pero estas posibilidades parecen estar esbozadas por lo que se espera socialmente, sin embargo, Ilama la atención que se carezca de un plan claro o estructurado para lograrlo, y que consideran que contar con una posición económica favorable, es lo que ayuda a cumplir el sueño:

"Uy! Yo siempre quise ser futbolista... A mí me gusta eso, uno ahí todo Cristiano (Ronaldo), con harta plata, así todo pinta y ese man es culo de jugador...ese man es muy bueno.- Y ¿cómo has hecho para ser futbolista?- Como así? ¿Que si he hecho algo para ser futbolista? -Si- pues jugar...en la cancha y eso... pero eso...eso es para pelados con suerte. A uno no le va a llegar aquí el man que lo recluta...no... eso no pasa, uno juega y se hace su fama en el barrio... Pero ya. Por eso le toca trabajar a uno cargando en la galería.- Ya, ¿crees que los chicos de estratos más altos tienen más posibilidades para hacer sus sueños como ser futbolista? - Si claro, eso es así, a eso no hay que darle vuelta. Que los papás los meten ahí a una escuela de futbol y ya está, si la man es bueno pues ahí queda reseñado. Y que les pagan la universidad y listo". (Hombre adolescente, Armenia) 


\subsection{De la familia a los amigos y la pareja: Transformaciones de los vínculos} sociales y nuevos contextos de interacción

Frente a la relación con la familia, se encuentra una diferencia significativa en ambas ciudades y esta está basada en la dimensión de la actitud; en Armenia los adolescentes reconocen que las decisiones y acciones que los padres tomen frente a ellos están orientadas a contribuir positivamente en su proceso de formación, pero mencionan que de igual forma buscan ganar mayor autonomía frente a los padres, por lo cual se genera rebeldía. Por su parte en Medellín hay dos asuntos, el primero es que frente a la familia aparece la rebeldía, la cual puede ser originada como respuesta a una imposición o por manifestación de desacuerdos; lo segundo es que los adolescentes mencionan que las relaciones con la familia pueden reemplazarse con los amigos cuando ellos están ausentes, pero cuando la familia está presente entonces se empieza a dejar secuencialmente el lugar de esta y se da paso a los amigos, sin que la familia deje de cumplir un rol fundamental, pero considerando que ya no se establece con ella una relación de obediencia sino una más caracterizada por el apoyo y la horizontalidad. Sin embargo, un factor común es que la familia no deja de perder autoridad en la vida del adolescente, y que la rebeldía tensiona la relación, pero la mueve en términos de ganar espacios de libertad y buscar espacios de búsqueda para el adolescente.

"El respeto, yo creo que a mí la adolescencia pues, a mí en parte, con algunas personas crece, con otras como que uno ya va bajando como el respeto, pero es como parte también como de la rebeldía, ¿no? Por ejemplo, con mis papás yo antes les tenía mucho respeto, no me atrevía a decir nada, pero ya como que no les tengo el mismo respeto que les tenía antes". (Mujer adolescente, Medellín)

"Pues pienso que esa etapa en vez de separarnos nos ha unido más, porque digamos después de cada discusión que tengamos o de cada desacuerdo o alguna cosa, vamos a salir más fuertes de eso, y es así, o sea, es como... es que... yo veo que cuando discuten como de una manera tan pendeja, es que es como uno se agarra a... uno tiene que estar pendiente de que todo lo que hacen los papás es por el bien de uno, así uno no le guste o así uno piense que eso está mal, los papás hacen todo por el bien de uno". (Mujer adolescente, Armenia)

"(los papás) Que usted por qué hace tal cosa, que por ejemplo si uno hace a uno lo regañan por hacer tal cosa, uno no hace esa cosa, 
iah! por qué no la hizo!... y es así... muy maluco!... pues mi mamá decía que solo me aguantaba ella ...Además ellos ya pasaron por esa etapa entonces ya han vivido cosas que nosotros no, que apenas vamos a vivir, entonces quieren como cuidarnos de esas cosas que nos pueden pasar...Porque... yo creo que uno necesita... digamos... como una voz superior que le diga a uno lo que está mal". (Hombre adolescente, Armenia)

Con relación a los amigos, en Medellín los adolescentes mencionan que los de la niñez son diferentes a los actuales ya que aquellos se basaban en relaciones de juego, mientras que con los de ahora se establece un vínculo estrecho y duradero que se escoge, y que se basa en funciones de diversión, corrección, seguridad, apoyo, aceptación, toma de decisiones e influencia, las mismas que se asignan a la familia:

"Esas amigas que solamente eran para chismosear en el corredor del colegio, ya ahora son las personas que te ayudan a tomar decisiones, que te dicen como "ino, tú sí puedes y vas a lograr esto en la vida!", que eso es lo que, desde que tú naces, son los papás, entonces por decir, mis papás me corregían, me decían como, "ino, no puedes hacer esto!", y ahora mis papás y mis amigas me lo dicen, entonces es como que, se vuelve muy similar, entonces yo digo que los amigos son la familia que uno escoge y si uno escoge esa familia es porque uno se siente demasiado cómodo con lo que es, entonces uno también busca en los amigos lo que no ve en la casa". (Mujer adolescente, Medellín)

En Armenia, los amigos se entienden como aquellos con los que se crea un vínculo emocional, se viven momentos inolvidables que marcan la vida y con los que se comparten gustos, secretos y salidas. Se convierten en aquellos en los que se confía, guían el proceso de la adolescencia e influyen en los cambios de gustos y actividades, además, se dividen en aquellos que te llevan por el mal camino y aquellos que te llevan por el buen camino. Marcan una relación diferenciada con la familia, pues los amigos permiten un espacio de confianza para compartir experiencias y opiniones que no se consideran objeto de discusión con los padres:

"Pues, en general... yo tengo... la mayoría de mis amigos son fotógrafos... entonces tomamos fotos y así, vamos al parque de la vida y tomamos fotos allá, patinamos o hacemos alguna cosa y otras veces vamos a centros comerciales, pero vamos muy poquitas veces 
porque pues, son demasiadamente llenos y de pronto nos roban. Vamos a comer, icomer helado y ya!, pues... hablar un rato y nos vamos a cine, de vez en cuando" (Mujer adolescente, Armenia).

"Mi tipo de amigos que yo sé que son amigos de toda la vida... es muy buena... son como mis hermanos, pero, o sea... somos muy tranquilos en sí... y somos como así todos ... no se sienten... ninguno tiene malos vicios, ni nada... somos muy recocheros y a veces muy alocados, pero, pues ...no tenemos malos vicios... con la relación con personas que sí han tocado como con esos temas... con la drogadicción y esas cosas... obviamente uno no los puede rechazar porque son personas ... como aquellas personas que dicen que dime con quién te juntas y te diré quién eres... y uno juntarse con una persona así es como medio maluco, de pronto se dan situaciones que ... que uno no puede controlar, ambientes que son feos, entonces mejor como evitar eso". (Mujer adolescente, Armenia)

En cuanto a las relaciones de noviazgo, en Medellín tienen una alta importancia y están emparejadas con las relaciones sexuales, a lo cual, según los participantes de menor edad, llegarán cuando sean "grandes", es decir, en las fases finales de la adolescencia:

"Ya después pasaría como al tema de tener la novia, de empezar con las relaciones sexuales, y así, y pues todavía para mí no, eso llegará más adelante algún tiempo. Creo que todavía, la mayoría de sentimientos vendría a ser que es el amor cuando, ese es como el primer amor serio que uno tiene que vendría a ser los 15, 16 años que eso sí dura un poquito más que todos los que usted ha tenido". (Hombre adolescente, Medellín)

En Armenia, se da una diferencia entre el noviazgo oficial y aquel que es considerado como "los que le caen", son estos amores con quienes se experimenta el romanticismo, el erotismo y la sexualidad. Se ve una marcada diferencia entre la visión de los hombres y las mujeres, pues ellas piensan más en la pareja ideal y ellos en lo físico.

"Sí, obvio... en la etapa aparecen mucho que el novio, que la novia, que te conquista, que te cae, que todo eso... pero pues, uno tiene que como, saber qué intenciones tiene la persona con uno, así como decía ella, no quedarse solamente con lo que le decían a uno, sino como indagar". (Mujer adolescente, Armenia) 
Todo lo anterior permite considerar que el campo representacional de la RS sobre adolescencia en Medellín y Armenia, comparte elementos comunes en las dimensiones de la información y la actitud, los cuales se relacionan con definirla como una etapa en la que se establece una relación diferente con la cotidianidad, donde se experimentan cambios en varias dimensiones, se esboza o establece una pregunta sobre el futuro y se asumen responsabilidades; es también el momento de la vida en el cual se construyen respuestas sobre quién se es y hacia dónde se dirige. No obstante, hay elementos que marcan diferencias sutiles en la forma de vivir y significar la adolescencia; dichas diferencias están relacionadas con las posibilidades que el contexto ofrece a los adolescentes.

\section{DISCUSIONES}

Con base en la información recolectada en dos ciudades de Colombia, Medellín y Armenia, con características sociodemográficas diferentes, a adolescentes entre los 12 y 18 años, se logra comprender cómo se estructura, organiza y ancla la representación social existente sobre adolescencia, desde y en, los adolescentes mismos.

A lo largo de este proceso fue posible definir ciertas diferencias y similitudes destacables entre ambas ciudades después del análisis de la información generada, lo que llevó a identificar algunas categorías que, con algunas sutilezas de tipo cualitativo, permiten afirmar que los procesos de objetivación y anclaje de las representaciones sociales entre ambas ciudades se encuentran y desencuentran en ciertos puntos.

Lo que parece configurar la semejanza más clara obtenida en las dos ciudades, es lo que se denominó núcleo figurativo en ambas, pues la representación social sobre adolescencia parece girar alrededor de la idea según la cual esta es una transición. En Medellín se concibe la adolescencia como un proceso en el cual se abandona la niñez y se prepara para la adultez; por su parte, en Armenia esta transición implica un proceso que se vive antes de ser adulto y que se narra como algo "normal" que hace parte del desarrollo de todas las personas. Además de esta semejanza, se encontró que en ambas ciudades se resaltan los cambios emocionales y físicos como características esenciales de la adolescencia que llevan hacia una búsqueda de sí mismos; de forma similar, se describen las relaciones con los amigos, tomándolos como aquellos que brindan apoyo y con los cuales se forman lazos perdurables, y que al mismo tiempo ejercen influencia sobre ellos. 
Otra semejanza que se consideró como importante para tener en cuenta, es el impacto que tiene el estrato socioeconómico sobre la concepción de adolescencia y sobre la visión que los adolescentes construyen del mundo y de su futuro, ya que, según los resultados de la investigación, los participantes dan cuenta de unas limitaciones en la construcción del futuro relacionadas con un menor estrato socioeconómico. Además, el contexto en el que se encuentran los adolescentes durante su desarrollo es de gran importancia ya que esto deja una huella en la visión que tienen frente al final de la adolescencia, la manera en que viven la sexualidad e incluso lo que implica "convertirse" en adulto en ambas ciudades.

$Y$ es precisamente este punto el que empieza a marcar las diferencias entre las dos ciudades. Por su lado, Medellín, al ser una ciudad más cosmopolita y en donde el tema del desarrollo económico y social moviliza a la ciudad, a las políticas públicas y a las personas en general (Pineda, 2016) parece llevar a los adolescentes a pensar con mayor empeño y afán en el futuro y en el fin de la adolescencia, creando una idea de una etapa en la que se pasa de una cosa para otra, donde se alcanza la adultez como fin último y en la que se adquieren deberes y responsabilidades. Entre tanto, en Armenia, al ser una ciudad más pequeña, más ruralizada y con un desarrollo social y económico más lento y con algunas dificultades en cuanto a desempleo e informalidad (DANE, 2016), la adolescencia se piensa si bien como una transición, en cuanto a verse a sí mismo como alguien diferente, el tema del futuro y el fin de la adolescencia no parece ser tan claro para estos adolescentes. Lo anterior es evidente en la ambigüedad y borrosidad con la que se presentan estos asuntos, pues el adolescente hace alusión al futuro sin tener un plan específico, cosa que sí ocurre en Medellín.

Además de este punto, que se presenta como discrepante entre ambas ciudades, el concepto de cambios psicológicos, la forma de relacionarse con los padres, el tema de las relaciones amorosas y la sexualidad también lo son, pues se encontró que se denominan de manera diferente. En Armenia, los cambios psicológicos se traducen en la necesidad de búsqueda de sí mismo, de vivir, experimentar y lograr aprendizajes; mientras que, en Medellín, se habla de los cambios en la posición frente a la vida, de tener sueños, darse cuenta, perder la inocencia y cuestionarse. Si se quiere, podría puntualizarse en que quizás el adolescente de Medellín si bien se busca a sí mismo, construye su identidad, está en relación con el escenario social con mayor fuerza que lo que determinan y proponen los adolescentes de Armenia, quienes parecen llevar un proceso más individual y volcado hacia sí mismos, quizá influen- 
ciado por las mismas características contextuales de las ciudades. Esto último estaría en relación con lo propuesto por Martuccelli (2007), quien recuerda que el individuo desde edades más tempranas reconoce poca sujeción a las estructuras sociales.

Frente a la relación con la familia, en Armenia los adolescentes reconocen que las decisiones y acciones que los padres tomen frente a ellos están orientados a contribuir positivamente en su proceso de formación, pero mencionan que de igual forma buscan ganar mayor autonomía frente a estos, por lo cual se genera cierta rebeldía. Por su parte en Medellín aparecen dos asuntos, el primero de ellos es que frente a la familia aparece la rebeldía, la cual puede ser por una imposición o manifestación de desacuerdos; lo segundo es que los adolescentes mencionan que las relaciones con la familia pueden remplazarse con los amigos cuando ellos están ausentes, se da paso a los amigos porque la familia empieza a tomar el rol de autoridad, generando lo que habitualmente se conoce como brecha generacional (Barrera, 2010).

Por otro lado, se evidencian diferencias en cuanto al impacto que generan los estratos socioeconómicos en ambas ciudades; Medellín es una ciudad en la cual los estratos socioeconómicos tienen mayor importancia, por lo que los adolescentes sienten que es diferente crecer en un estrato socioeconómico alto que en uno bajo. Por su parte, en Armenia, no se ve tan marcada la diferencia entre los estratos socioeconómicos, pues dentro de los estratos bajos hay familias con altos niveles de educación, sin embargo, se encontró que los jóvenes con mayor capacidad económica tienen acceso a más oportunidades que los llevan a pensar con mayor facilidad en un futuro por lo que se concluye que el ambiente en el cual se desarrolla la adolescencia es diferente dependiendo del estrato. Este aspecto resulta interesante en la medida que se acerca a lo que algunos autores vienen defendiendo y es la idea de que la adolescencia es una construcción social, más que biológica y por tanto la manera en que se vive y se representa está marcada por esas condiciones del contexto y la época histórica (Zanotto, 2012). Por último, en cuanto al final de la adolescencia, se aprecia una diferencia significativa; en Medellín se enmarca más en términos de responsabilidad frente a sí mismo y madurez, mientras que en Armenia se hace referencia a la independencia, al logro de irse de la casa.

Teniendo en cuenta lo descrito, y para poder hablar de los procesos de objetivación y anclaje de la representación social construida por los adolescentes de dichas ciudades, se debe hacer alusión al núcleo figurativo que se dibuja 
en ambas, el cual parece estar centrado, como ya se dijo, en que la adolescencia es una transición, una en la que se da la búsqueda de sí mismo, y por tanto la adolescencia es objetivada desde allí como una etapa de transición que por demás parece ser normal.

Sin embargo, tal objetivación está más centrada, en la ciudad de Armenia, en la experimentación de nuevas sensaciones, emociones, situaciones, y pensamientos, mientras que, en la ciudad de Medellín, si bien se busca experimentar, lo que se busca es sobre todo, conocer del futuro, dado que el fin último de la transición es ser adulto y tener responsabilidades. Con lo anterior, cobra validez y sentido el hecho de proponer que el anclaje para las dos ciudades está dado desde las condiciones contextuales y las posibilidades y lógicas en las que se desenvuelven ambas ciudades, que como ya se mencionó son diversas.

A partir de la información recolectada podría analizarse el impacto que esto tiene en la vida cotidiana y la relación con la teoría existente de la concepción de adolescencia. Según los resultados encontrados, no existen diferencias significativas a nivel estructural, es decir, el contenido de la adolescencia es igual que la teoría en tanto ambos hablan de cambios físicos, emocionales, familiares, de amigos, en la toma de decisiones; sin embargo, a la hora de profundizar en el proceso de constitución de la Representación Social, la objetivación y el anclaje muestran elementos sutiles pero que generan formas de concebir la adolescencia de manera diferente. Constatándose, una vez más que son aquellos elementos periféricos los que parecen ordenar y dar sentido a los elementos nucleares, tal como lo propone Moliner (2007).

En este sentido queda claro que las representaciones sociales, si bien tienen un componente estructural y cognitivo, están permeadas por el contexto y época histórica convirtiéndose también en un proceso de construcción social, a través del cual los sujetos plasman en sus ideas y actitudes todo aquello que viven y lo que los permea, creándose a su vez una relación estrecha entre las condiciones objetivas y las subjetivas (Rodríguez, 2007).

Para cerrar, queda la pregunta abierta para una futura profundización de la influencia de las amistades en la concepción de la adolescencia y la forma en la que esta se desarrolla al ser considerada por los participantes como una parte esencial de esta etapa; también queda abierta la pregunta respecto a la concepción que los adultos tienen sobre esta etapa. Se podría profundizar más en futuras investigaciones sobre este aspecto. Así mismo, es importante 
continuar adelantando investigaciones que se ocupen de la forma en que las condiciones del contexto atraviesan la construcción de proyectos de vida y la concepción de futuro; esto puede contribuir a la construcción de procesos de intervención y formulación de políticas públicas con la población de adolescentes, en los cuales se pase de miradas centradas en la transmisión de información sobre la etapa misma y los cambios que se experimentan a fin de reducir los riesgos que a ella se asocian, a unas que le permitan al adolescente reflexionar sobre las experiencias que vive y tomar decisiones frente a ellas fundamentadas en las concepciones que tienen acerca de su propia vida y de la forma en que quieren construirla, pero también analizar las oportunidades que puede ofrecer el contexto para que el adolescente logre proyectarse en futuros posibles.

Investigaciones como estas son un aporte a la generación de conocimiento contextualizado de la adolescencia, lo que puede ayudar a incrementar las posibilidades para que se actúe desde la política pública en beneficio de esta población y de su constructo social, así como puede ayudar a las familias y adultos en general a comprender el universo en el que se mueve el adolescente y hacerlo partícipe activo de su propia vida en esa cosmovisión de imágenes, ideas, concepciones y versiones en las que él es el epicentro de muchos debates.

Agradecimiento: A la Corporación Universitaria Alexander Von Humboldt y a la Universidad CES por todo el apoyo administrativo y académico en la realización de la investigación; a los estudiantes de pregrado del semillero de investigación que contribuyeron al proceso de generación de la información; a los adolescentes que amablemente aceptaron colaborar con el proceso.

Financiación: Universidad CES, Medellín. Representaciones sociales sobre adolescencia construidas por adolescentes y adultos en las ciudades de Medellín y Armenia. INV022015017. Corporación Universitaria Alexander Von Humboldt. Representaciones sociales sobre adolescencia construidas por adolescentes y sus adultos significativos en las ciudades de Medellín y Armenia. D.I.P. 04-2015

\section{REFERENCIAS}

Acero, A. R., Escobar, F., \& Castellanos, G. (2007). Factores de riesgo para violencia y homicidio juvenil. Revista Colombiana de Psiquiatría, 26(1), 78-97. Recuperado de http://www.scielo.org.co/pdf/rcp/v36n1/v36n1a07.pdf

Alba, L. H. (2010). Salud de la adolescencia en Colombia: bases para una medicina de prevención. Revista Universitas Médica. Bogotá (Colombia), 51(1). 29-42. Recuperado de https://www.redalyc.org/articulo.oa?id=231018676001

Alcaldía de Armenia. (2014). Política Pública de Juventud de Armenia "Jóvenes Construyendo Ciudad" 2014 - 2024 [Versión electrónica]. Recuperado de http:// 
www.armenia.gov.co/wp-content/uploads/2016/12/Pol\%C3\%ADtica_P\%C3\%BAblica_de_Juventud_2014.pdf

Alcaldía de Medellín (2014). Política Pública de Juventud de Medellín [Versión electrónica]. Recuperado de https://www.medellin.gov.co/irj/go/km/docs/ pccdesign/SubportaldelCiudadano_2/PlandeDesarrollo_0_10/ProgramasyProyectos/Shared\%20Content/Documentos/2015/Política_PúblicadeJuventud_ Medellín.pdf

Alcaldía de Medellín (2015). Perfil demográfico 2016-2020 [Versión electrónica]. Recuperado de https://www.medellin.gov.co/irj/go/km/docs/pccdesign/SubportaldelCiudadano_2/PlandeDesarrollo_0_17/IndicadoresyEstadsticas/Shared\%20 Content/Documentos/ProyeccionPoblacion2016-2020/Perfil\%20Demogr\%C3\%A1fico\%202016\%20-\%202020\%20Total\%20Medellin.pdf

Alpízar, L., \& Bernal, M. (2003). La Construcción Social de las Juventudes. Última década, 11(19), 105-123. https://doi.org/10.4067/S0718-22362003000200008

Andrade, J. A. (2012). Aspectos psicosociales del comportamiento suicida en adolescentes. Revista Electrónica de Psicología Iztacala. 15, (2). 688-721. Recuperado de http://www.revistas.unam.mx/index.php/repi/article/view/32373/29770

Araya, S. (2002). Las Representaciones Sociales: Ejes teóricos para su discusión. Costa Rica: Facultad Latinoamericana de Ciencias Sociales (FLACSO). Recuperado de http://unpan1.un.org/intradoc/groups/public/documents/ICAP/UNPAN027076. pdf

Banchs, M. (2000). Aproximaciones Procesuales y Estructurales al estudio de las Representaciones Sociales. Papers on Social Representation, 9, 3.1-3.15. Recuperado de http://psr.iscte-iul.pt/index.php/PSR/article/view/269/234

Barrera, D. (2010). Empujes y resistencias al cambio en familias con hijos adolescentes. Boletín de Antropología Universidad de Antioquia, 24(41), Medellín, 376-394. Recuperado de http://bibliotecadigital.udea.edu.co/bitstream/10495/2337/6/ BarreraDagoberto_2010_EmpujesResistenciasCambio.pdf

Becoña, E. (2000). Los adolescentes y el consumo de drogas. Papeles del psicólogo, 77, 25-32. Recuperado de https://www.redalyc.org/html/778/77807705/

Blázquez, M. S. (2012). Embarazo adolescente. Revista Electrónica Medicina, Salud y Sociedad, 3 (1), 1-8. http://dx.doi.org/10.25009/remsys.2012.1.98

Bonilla, E., \& Rodriguez, P. (1997). Manejo de datos cualitativos. En E. Bonilla, \& P. Rodriguez, Más allá del dilema de los métodos. pp.131-168. Bogotá: Ediciones Uniandes.

Brunet, I., \& Pizzi, A. (2013). La delimitación sociológica de la juventud. Ultima década, 21(38), 11-36. https://doi.org/10.4067/S0718-22362013000100002.

Carrillo, G., De la Rosa Bobadilla, J. J., \& Gutiérrez, A. M. (2014). Ámbitos de Actuación del Trabajo social y su aporte al desarrollo en el departamento del Quindío. (Informe final de investigación). Universidad del Quindio, Armenia Colombia.

Chaves, M. (2005). Juventud negada y negativizada: Representaciones y formaciones discursivas vigentes en la Argentina contemporánea. Última Década, 23, 9-32. Recuperado de https://www.redalyc.org/articulo.oa?id=19502302

Congreso de Colombia (2006). Ley 1098 de 2006, por medio de la cual se expide el código de la infancia y la adolescencia. Recuperado de https://www.oas.org/dil/ esp/Codigo_de_la_Infancia_y_la_Adolescencia_Colombia.pdf

Consejo Municipal de Política Social y de Equidad, A. (Diciembre de 2014). Obtenido de Políticas Poblacionales para la Vida y la Equidad: https://www.medellin. gov.co/irj/go/km/docs/pccdesign/SubportaldelCiudadano_2/PlandeDesa- 
rrollo_0_17/Publicaciones/Shared\%20Content/Documentos/2015/LIBRO\%20 -\%20POLITICAS\%20POBLACIONALES\%20digital\%20media.pdf

DANE (2016). Boletín técnico 2015. Principales indicadores del mercado laboral. Bogotá: DANE. Recuperado de https://www.dane.gov.co/files/investigaciones/ boletines/ech/ech/bol_empleo_dic_16.pdf

Deossa Restrepo, G. C., Restrepo Betancur, L. F., \& Velásquez Vargas, J. E. (2015). Actitudes y comportamientos de riesgo para trastornos de la conducta alimentaria en jóvenes universitarios, Colombia. Hacia la Promoción de la Salud, 20(1), 67-82. Recuperado de http://www.scielo.org.co/pdf/hpsal/v20n1/v20n1a05.pdf

Denzin, N. K., \& Lincoln, Y. S. (2011). Introducción general. La investigación cualitativa como disciplina y como práctica. En Manual de investigación cualitativa (Primera edición, Vol. 1, pp.43-101). Barcelona: Gedisa.

Gobernación del Quindío. (2016). Departamento del Quindío Proyecto de Ordenanza, plan departamental de desarrollo 2016-2019. Recuperado de http://quindio.gov. co/medios/imagenes/PLAN_DE_DESARROLLO_2016-2019.pdf

Gobierno del Quindío. (2012). Plan de desarrollo departamental 2012-2015 “Gobierno firme por un Quindío más humano". Recuperado de https://www.quindio.gov.co/ home/docs/items/item_100/PLAN\%20DE\%20DESARROLLO\%202012-2015\%20 FINAL.pdf

González, J. C. \& Andrade, J. A. (2012). Asociación entre el consumo de sustancias psicoactivas y actividad delictiva en adolescentes. Poiesis, 24, 1-10. https://doi. org/10.21501/16920945.314

Hamui-Sutton, A., \& Varela-Ruiz, M. (2013). La técnica de grupos focales. Revista de Metodología de Investigación en educación médica, 2(1), 55-60. Recuperado de http://www.scielo.org.mx/pdf/iem/v2n5/v2n5a9.pdf

Jodelet, D. (1986). La representación social: fenómenos, concepto y teoría. En: S. Moscovici, editor. Psicología social II. Barcelona: Paidós. pp.470-94.

Limones, I. (2011). Adolescentes y percepción del sí mismo: la construcción de una imagen realista de la adolescencia desde la familia y la escuela. Etic@net, 9(11), https://dialnet.unirioja.es/servlet/articulo?codigo=3802191

Loboa, N., \& Morales, D. (2013). Perfil de orientación al suicidio en adolescentes escolarizados, Villahermosa - Tolima, 2013. Facultad Nacional de Salud, 34(1), 94-102. Recuperado de https://aprendeenlinea.udea.edu.co/revistas/index.php/ fnsp/article/view/19885/20779294

Luna Bernaly, A., \& Cruz Abundis, C. (2014). Frecuencia e intensidad de conflictos con los padres en adolescentes bachilleres. Alternativas en Psicología, 18(30), 8-21. Recuperado de http://alternativas.me/attachments/article/51/1\%20 -\%20Frecuencia\%20e\%20intensidad\%20de\%20conflictos\%20con\%20los\%20 padres\%20en\%20adolescentes\%20bachilleres.pdf

Margulis, M. (2004a). ¿Juventud o juventudes? Perspectiva, 22(2), 297-324. Recuperado de https://periodicos.ufsc.br/index.php/perspectiva/article/ viewFile/9644/8873

Margulis, M. (2004b). Adolescencia y Cultura en la Argentina. Perspectivas Metodológicas, 1(4), 1-7. http://dx.doi.org/10.18294/pm.2004.574

Martuccelli, D. (2007). Cambio de rumbo. La sociedad a escala del individuo. Chile: LOM Ediciones.

Materán, A. (2008). Las representaciones sociales: un referente teórico para la investigación educativa. Geoenseñanza, 13(2), 243-248. Recuperado de http://www. redalyc.org/pdf/360/36021230010.pdf 
McKinney, J. P., Fitzgerald, H., \& Strommen, E. (1993). Psicología del desarrollo: edad adolescente. México: El Manual Moderno.

Moliner, P. (2007). La teoría del núcleo matriz de las Representaciones Sociales. En T. Y. Rodríguez, Representaciones Sociales. Teoría e investigación (pp.137-156). Guadalajara; México: CUCSH-UDG.

Moscovici S. (1963). Attitudes and opinions. Annu Rev Psychol, (14), 231-260. https:// doi.org/10.1146/annurev.ps.14.020163.001311

Moscovici S. (1979). El psicoanálisis, su imagen y su público. Argentina: Huemul.

Papalia, D. E., Olds, W. S., de León, E. P., \& Villamizar, J. (1997). Psicología del desarrollo: de la infancia a la adolescencia. México: McGraw-Hill.

Pineda, H. (2016). Clúster, política de internacionalización y promoción. En Contexto, 4(5), 83-98. Recuperado de http://ojs.tdea.edu.co/index.php/encontexto/article/ view/366/383

Pavez, I (2012). Sociología de la infancia: las niñas y los niños como actores sociales. Revista de sociología, 27, 81-102. Obtenido de de https://revistadesociologia. uchile.cl/index.php/RDS/article/view/27479/29152

Ridao, P., \& Moreno, C. (2008). Percepción de los adolescentes y sus progenitores de la adolescencia como etapa evolutiva. Infancia y Aprendizaje, 31(4), 499-513. Recuperado de https://idus.us.es/xmlui/bitstream/handle/11441/32150/ Percepci\%F3n\%20de\%20los\%20adolescentes\%20y\%20sus\%20progenitores. pdf?sequence $=1$

Rodríguez, T. (2007). Sobre el estudio cualitativo de la estructura de las Representaciones Sociales. En T. Y. Rodríguez, Representaciones Sociales. Teoría e investigación (pp.157-189). Guadalajara; México: CUCSH-UDG.

Salamanca, A, \& Martín-Crespo, C. (2007). El muestreo en la investigación cualitativa. Nure Investigación, (27), 1-4. Obtenido de http://ceppia.com.co/Documentos-tematicos/INVESTIGACION-SOCIAL/MUESTREO-INV-CUALITATIVA.pdf

Santrock, J. (2004). Psicología del desarrollo en la adolescencia. Madrid: McGraw-Hill.

Schindler Andino. (4 de marzo de 2013). Medellín la ciudad más innovadora del mundo. Obtenido de http://www.schindler.com/co/internet/es/sobre-schindler-andino-colombia/noticias/2013/medellin-la-ciudad-mas-innovadora-delmundo.html

Secretaría del Concejo Municipal. (7 de junio de 2016). Plan de desarrollo "Medellín cuenta con vos" 2016-2019. Obtenido de https://www.medellin.gov.co/irj/go/ km/docs/pccdesign/SubportaldelCiudadano_2/PlandeDesarrollo_0_17/Publicaciones/Shared\%20Content/Documentos/2016/GACETA\%204383.pdf

Silva, I. (2007). La adolescencia y su interrelación con el entorno. España. Recuperado de http://www.injuve.es/sites/default/files/LA\%20ADOLESCENCIA\%20y\%20\%20 entorno_completo.pdf

Taguenca, J. (2016). Sociología de la juventud. Una revisión. Espacio abierto, 25(3). Recuperado de http://www.redalyc.org/articulo.oa?id=12249678013

Tesouro i Cid, M., Palomanes Espadalé, M. L., Bonachera Carreras, F., \& Fernández Martínez, L. M. (2013). Estudio sobre el desarrollo de la identidad en la adolescencia. Tendencias psicológicas, (21), 211-224. Recuperado de https://revistas. uam.es/tendenciaspedagogicas/article/view/2034/2142

Toro, D. C., Paniagua, R.E., González, C.M., \& Montoya, B. (2009). Caracterización de adolescentes escolarizados con riesgo de suicidio, Medellín, 2006. Revista Facultad Nacional de Salud Pública, 27(3), 302-308. Recuperado de https:// aprendeenlinea.udea.edu.co/revistas/index.php/fnsp/article/view/1446/4198 
Vergés, P. (1992). L'evocation de L'argent: Une Méthode Pour La Définition Du Noyau Central D'une Représentation. Bulletin de Psychologie, 45 (405), 203-209. Recuperado de https://www.researchgate.net/publication/232451723_L'evocation_de_l'argent_Une_methode_pour_la_definition_du_noyau_ central_d'une_representation_The_evocation_of_money_A_method_for_ defining_the_central_core_of_a_representation

Wagner, W., Hayes, N., \& Flores, F. (2011). El discurso de lo cotidiano y el sentido común. México: Anthropos.

Zanotto, A. (2012). Apuntes sobre una representación social de la adolescencia en los editoriales del diario La Nación. Subjetividad y procesos cognitivos, (16), 245-264. Obtenido de http://www.scielo.org.ar.bdigital.ces.edu.co:2048/pdf/spc/v16n1/ v16n1a11.pdf 